Proceedings of the 5th International Symposium on Communications, Control and Signal Processing, ISCCSP 2012, Rome, Italy, 2-4 May 2012

\title{
PERCEPTUAL NON LOCAL MEAN (P-NLM) DENOISING
}

\author{
W. Souidene ${ }^{1}$, S. Megrhi ${ }^{1,2}$ \\ ${ }^{1}$ L2TI, 99 avenue J.B Clément \\ 93430, Villetaneuse \\ wided.mseddi, beghdadi@univ-paris13.fr
}

\author{
A. Beghdadi ${ }^{1}$, C. Ben Amar ${ }^{2}$ \\ ${ }^{2}$ Ecole Nationale d'Ingénieurs de Sfax, \\ Département de Génie Electrique \\ Route de Soukra, B.P. 1173, 3038, Sfax, Tunisie \\ chokri.benamar@ieee.org, sam.khanfir@gmail.com
}

\begin{abstract}
In this paper we propose a denoising technique based on nonlocal means using an image similarity measure. The idea is to use the SVD-based image quality metric as a measure of neighborhood similarity. This measure is then used in the computation of the spatial Gaussian weighting kernel. We also develop an optimization computation scheme using a parallel architecture in order to accelerate the filtering process on different machines or different cores on the same machine. The obtained results are very promising.
\end{abstract}

Keywords : image denoising, non local means filtering, perceptual model, perceptual filtering, objective image similarity.

\section{INTRODUCTION}

Image denoising is one of the most attractive research fields in image processing. A number of recent studies have shown that exploiting the spatial and photometric redundancy of the image in the design of the filtering process yield interesting results and overcome the classical approaches. This redundancy appears not only on some textured images but also in many natural images. The Non Local Mean (NLM) technique introduced in [1] restores the original image by considering non local neighborhoods of a given pixel. It has been shown that the concept of non local neighborhoods is very relevant for natural as well as textured images. In fact, it exploits the redundancy and allows a better contribution of different image structures to denoise similar ones. The notion of similarity is very often computed as an MSE-based measure which is not actually correlated with the human perception. In this article, we first present an overview of the NLM as introduced in [1] then we propose a different scheme of similarity calculation inspired by some characteristics of the on the Human Visual System (HVS). The basic idea is to use a more consistent measure of similarity between windows in order to select the most perceptual relevant windows in the weighting process. The filtering is then performed based on these selected windows. However, the improvement, in terms of denoising quality, is gained at the expense of complexity and time con- sumption. To this end, we propose an optimized and rapid implementation of the filtering based on a parallel architecture. The paper is organized as follows. Section 2 introduces the NLM concept and the a most important steps. The following section presents the proposed idea. Section 4 gives some details on the parallelization scheme implementation. The results are given in section 5. Finaly the last section is devoted to conclusion and perspectives.

\section{PROBLEM STATEMENT}

The Non Local Means denoising technique has been recently developed to overcome the limitations of classical linear filtering methods [2][1] [3][4][5]. Its principle is quite simple and it was used in the spatial domain as well as in the transform domain [6]. Using a noisy version $g$ of the original image $\mathbf{f}$, we restore each pixel using its neighbors in a prespecified region of the image. The definition of a neighbor is no longer a "spatial" definition but a definition based on similarity between pixels. So taht, pixels $i$ and $j$ will be considered are neighbors if they belong to similar structures of the image. Let $I$ be the support of original and noisy images (we suppose that they have the same size). Let $\left(n_{1}, n_{2}\right)$ be a pixel in noisy image $\mathbf{g}$. The estimated value of the pixel $\left(n_{1}, n_{2}\right)$ in the original image is computed as a weighted average of all the pixels in the image (or in a portion of the image):

$$
\mathbf{f}\left(n_{1}, n_{2}\right)=\sum_{\left(k_{1}, k_{2}\right) \in\left[1, m_{f}\right] \times\left[1, n_{f}\right]} w_{n_{1}, n_{2}}\left(k_{1}, k_{2}\right) \mathbf{g}\left(k_{1}, k_{2}\right)
$$

where $w_{n_{1}, n_{2}}\left(k_{1}, k_{2}\right)$ is a weight associated with the similarity between pixels $\left(n_{1}, n_{2}\right)$ and $\left(k_{1}, k_{2}\right)$.

$$
w_{n_{1}, n_{2}}\left(k_{1}, k_{2}\right)=\frac{1}{Z\left(n_{1}, n_{2}\right)} \exp \left(-\frac{\left\|\mathbf{g}_{n}-\mathbf{g}_{k}\right\|_{2}^{2}}{\gamma^{2}}\right)
$$

where $\mathbf{g}_{n}$ and $\mathbf{g}_{k}$ denote the neighborhoods of pixels $\left(n_{1}, n_{2}\right)$ and $\left(k_{1}, k_{2}\right)$ respectively and $\gamma$ is a constant that controls the decay of the exponential function. $Z$ is a normalizing factor 
which is, for each pixel, the sum of all exponential terms.

$$
Z(m, n)=\sum_{\left(n_{1}, n_{2}\right)} \exp \left(-\frac{\left\|\mathbf{g}(m, n)-\mathbf{g}\left(n_{1}, n_{2}\right)\right\|_{2}^{2}}{r^{2}}\right)
$$

Note that, the similarity between two neighborhoods or patches centered on two pixels is estimated in terms of squared error. This similarity measure does not take into account the structural information and is not consistent with the human visual perception. So we propose here to use a similarity measure that accounts for the perceptual images quality. It has been shown that SVD-based image quality metric proposed in [7] correlates well with the subjective evaluation of human.

\section{PERCEPTUAL NLM DENOISING ALGORITHM}

The use of SVD in the design of NLM is not new [8]. Here, we use the SVD-based image fidelity metric as a measure of perceptual similarity of image patches. Indeed, the SVD has been proven to be an efficient signal decomposition tool in terms of energy compactness. Furthermore, it is well suited for local analysis of image.

\subsection{Objective image quality measure based on HVS}

In [7], a new image quality metric based on SVD has been proposed. It is based on a local analysis of the image through overlapping blocks. The SVD of each block is computed in the original and degraded image. Then, by computing the difference between the respective singular values a local index of similarity is extracted. For a block $i$, the associated index is given by:

$$
D_{i}=\sqrt{\sum_{j=1}^{n}\left(s_{j}-\hat{s}_{j}\right)^{2}}
$$

Where $s_{j}$ are the singular values of the original block, $\hat{s}_{j}$ are the singular values of the distorted block and $n$ is the block size assuming a square block of size $(n * n)$. The global distortion measure is then derived from the $D_{i}$ values as follows:

$$
M=\frac{\sum_{i=1}^{p}\left|D_{i}-D_{m e d}\right|}{k^{2}}
$$

Where $D_{m e d}$ is the median value of the $D_{i}$ 's and $k$ is the number of processed blocks in the image. In order to have comparable values of $M$ with the other metrics, we propose to use the following measure :

$$
M_{s v d}=20 \log _{10}(M)
$$

A large value for $M_{s v d}$ is associated with high level of distortion. The $M_{s v d}$ metric has the advantage to be simple to implement. Its complexity is of order $O\left(n^{3}\right)$, but when applied to each block it becomes computationally reasonable. It does not assume any a-priori knowledge about the distortion neither the original image and it does not require any tuning of the parameters. For these advantages, the $M_{s v d}$ measure was studied and chosen to perform the perceptual denoising of images.

\subsection{Perceptual denoising based on $M_{S V D}$}

In the original version of the NLM algorithm, a 'distance' is computed between the different neighborhoods in order to determine their similarity and their contribution to the denoising of the current pixel. In this article, we propose to replace this distance by an objective similarity measure based on the local energy of the signal. . Namely, the proposed expression of the NLM criterion becomes :

$\mathbf{f}_{S V D}\left(n_{1}, n_{2}\right)=\sum_{\left(k_{1}, k_{2}\right) \in\left[1, m_{f}\right] \times\left[1, n_{f}\right]} w_{n_{1}, n_{2}}^{S V D}\left(k_{1}, k_{2}\right) \mathbf{g}\left(k_{1}, k_{2}\right)$

where $w_{n_{1}, n_{2}}^{S V D}\left(k_{1}, k_{2}\right)$ is a weight associated with the objective similarity between pixels $\left(n_{1}, n_{2}\right)$ and $\left(k_{1}, k_{2}\right)$.

$$
w_{n_{1}, n_{2}}^{S V D}\left(k_{1}, k_{2}\right)=\frac{1}{Z^{S V D}\left(n_{1}, n_{2}\right)} \exp \left(-\frac{\left\|\mathbf{S}_{n}-\mathbf{S}_{k}\right\|_{2}^{2}}{\gamma^{2}}\right)
$$

$\mathbf{S}_{n}$ and $\mathbf{S}_{k}$ are the vectors of singular values of a window of size $\left(m_{w}, n_{w}\right)$ defined around the pixel $n$ and $k$ respectively. $Z^{S V D}$ is the normalizing factor :

$$
Z(m, n)=\sum_{\left(n_{1}, n_{2}\right)} \exp \left(-\frac{\left\|\mathbf{S}(m, n)-\mathbf{S}\left(n_{1}, n_{2}\right)\right\|_{2}^{2}}{r^{2}}\right)
$$

This new expression of the NLM criterion ensures a better fidelity to the human perception of similarity between two pixels.

\section{PARALLEL IMPLEMENTATION OF P-NLM}

Image denoising algorithms in general, and NLM in particular, are time and computing ressource consuming. Therefore, during the last decades there has been an increasing interest in the development and the use of parallel algorithms in image processing. Parallel processing is implemented using several methods, some of these are data parallelism and instruction parallelism. Data parallelism implies the distribution of the data among the different processors in order for each of these to perform the same processing task. Instruction parallelism implies that each processor will perform a different task but all of them will perform these tasks in parallel. In our case we use the data parallelism in order, for the inner loops of our image denoising algorithm, to treat chunks of the image data in parallel. We have studied the inner loops dependencies and extracted the ones that does nots present any data dependencies. Those loops were the ones distributed to the different processors in order the achieve the parallelism. The results show that the processing time decreases significally without 


\begin{tabular}{|c|c|c|c|c|}
\hline Algorithm & Cameraman & Lena & Baboon & Pirate \\
\hline Perceptual NLM & 0.98 & 0.99 & 0.89 & 0.96 \\
\hline Classical NLM & 0.79 & 0.75 & 0.58 & 0.63 \\
\hline
\end{tabular}

Table 1. Objective image quality for denoised images using perceptual NLM and classical NLM.

\begin{tabular}{|c|c|c|c|c|}
\hline Image & Lena & cameraman & baboon & pirate \\
\hline Acceleration rate & 4.7 & 4.4 & 4.6 & 4.7 \\
\hline
\end{tabular}

Table 2. CT of parallized perceptual NLM algorithm

affecting neither the quality of the images nor the efficiency of the algorithm.

\section{EXPERIMENTAL RESULTS}

To perform image denoising, we use four images of size $512 *$ 512 , namely : lena, baboon, cameraman and pirate. The noisy images are altered using an additive white gaussian noise of variance $V=0.8$. We use a machine equipped with an Intel(R) Core(TM) i7 CPU 960 2x@3.20GHz with 12,0 Go of RAM. We evaluate the denoised image quality using the IUQM [9]. The denoising was performed, first in a sequential way, then, exploiting the different processors available in a parallel implementation.

\subsection{Perceptual quality of denoised images}

The sets of noisy/denoised images using perceptual NLM algorithm are shown on figures 1, 2, 3 and 4 respectively. We show in these figures the denoised images using both the sequential and the parallel algorithm. Basically, the denoising is performed the same way and the denoised images are identical. The gain is obtained on computation time. The objective quality of the denoised images measured using SSIM metric is shown on table 1. This table compares the performance of the perceptual NLM algorithm towards classical NLM. We can notice that when considering a perceptual method to measure similarity between two regions of the image, we can improve the denoising performance.

\subsection{Algorithm computational rapidity}

In order to decrease the computational time (CT) we propose a parallel implementation of our algorithm. The computational time results are summarized on table 2 .

\section{CONCLUSION}

In this article, we propose a method to perform image denoising using NLM algorithm based on a perceptual image quality metric. We also propose a fast implementation of our algorithm using a parallel architecture based on a double Core PC. The algorithm has been proven to provide good denoising performance quality and is three times faster than the sequential one. In a future work, we will develop the parallelism of our algorithm, we will study the different ways to avoid boarder effect and we plan to use it for HD images.

\section{REFERENCES}

[1] A. Buades, B. Coll, and J.M. Morel, "A non-local algorithm for image denoising," in Proc. of the IEEE Conference on Computer Vision and Pattern Recognition, June 2005, vol. 2, pp. 65-66.

[2] A. Buades, B. Coll, and J.M. Morel, "The staircasing effect in neighborhood filters and its solution," IEEE Transactions on Image Processing, vol. 15, no. 6, pp. 14991505, June 2006.

[3] M. Elad and M. Aharon, "Image denoising via sparse and redundant representations over learned dictionaries," IEEE Trans. on Image Processing, vol. 15, no. 12, Jan. 2006.

[4] M. Protter and M. Elad, "Image sequence denoising via sparse and redundant representations," IEEE Trans. on Image Processing, vol. 18, no. 1, Jan. 2009.

[5] T. Thaipanich, B. T. Oh, P.-H. Wu, and C.-C. J. Kuo, "Adaptive nonlocal means algorithm for image denoising," in Digest of Technical Papers International Conference on Consumer Electronics ICCE, 2010, pp. 417-418.

[6] W. Souidene, A. Beghdadi, and K. Abed-Meraim, "Image denoising in the transformed domain using non local neighborhoods," in Proc. ICASSP, 2006, vol. 2, pp. 869872.

[7] A. Shnayderman, A. Gusev, and A. M. Eskicioglu, "A multidimensional image quality measure using singular value decomposition," in Proc. of the SPIE Image Quality and System Performance Conference, Jan. 2004, vol. 5294, pp. 82-92.

[8] T. Thaipanich, T. O. Byung, P-H Wu, D. Xu, and C.-C.J. Kuo, "Adaptive nonlocal means algorithm for image denoising," IEEE Trans. On Consumer Electronics, vol. 56, no. 4, pp. 2623 - 2630, November 2010.

[9] Z. Wang, E.P. Simoncelli, and A.C. Bovik, "Multi-scale structural similarity for image quality assessment," in Proc. IEEE Asilomar Conference, Nov. 2003. 

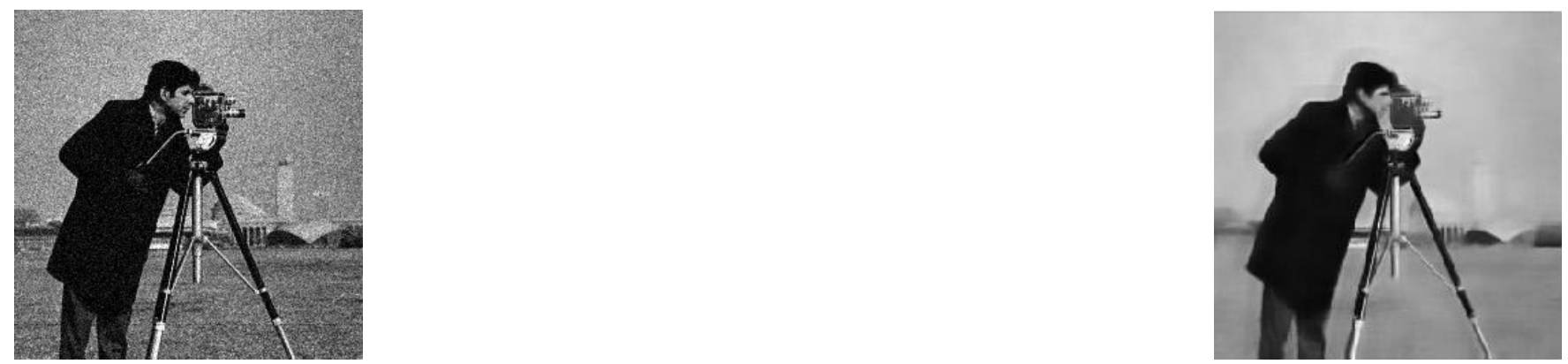

Fig. 1. (a) : $512 * 512$ noisy image ( $V=0.8$ ), (b) Denoised using sequential P-NLM
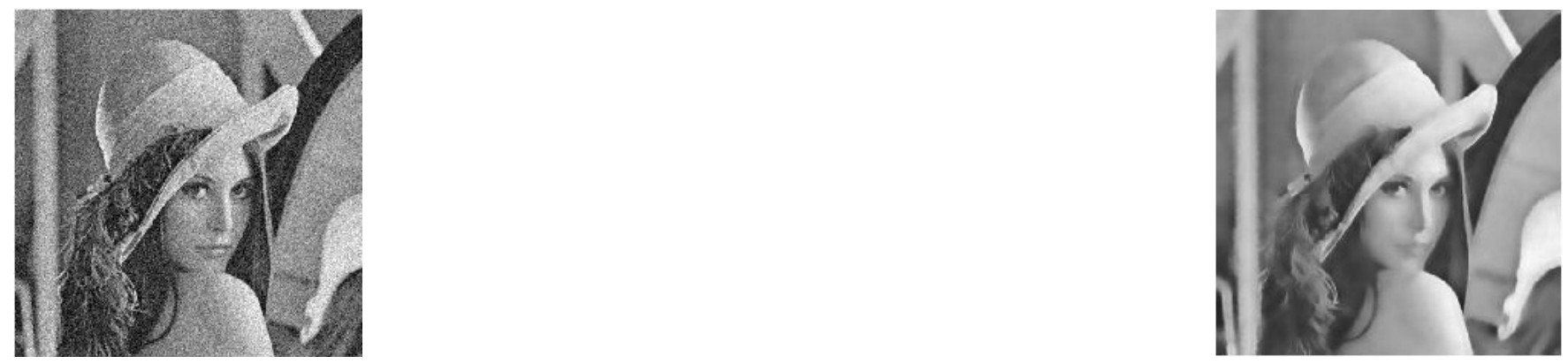

Fig. 2. (a) : $512 * 512$ noisy image ( $\mathrm{V}=0.8$ ), (b) Denoised using sequential P-NLM
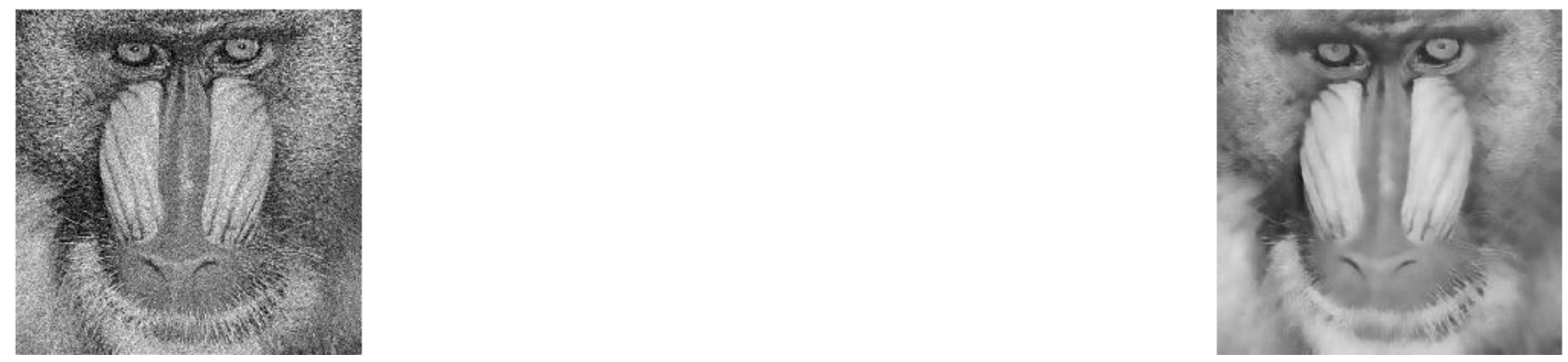

Fig. 3. (a) : $512 * 512$ noisy image ( $\mathrm{V}=0.8$ ), (b) Denoised using sequential P-NLM
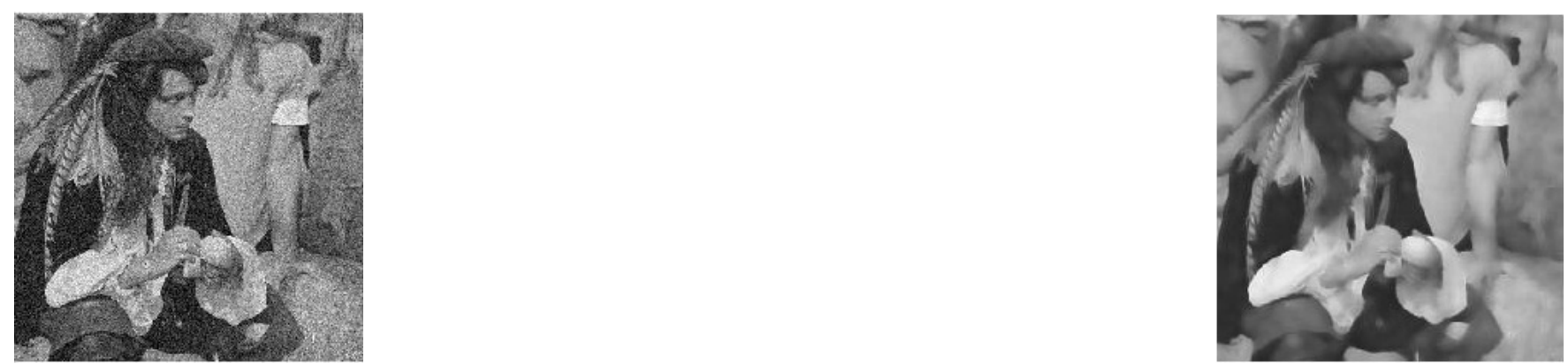

Fig. 4. (a) : $512 * 512$ noisy image ( $\mathrm{V}=0.8$ ), (b) Denoised using sequential P-NLM 